\title{
Dinámica grupal y toma de conciencia de la identificación proyectiva en estudiantes de Psicología
}

\author{
Group dynamic and awareness of the projective identification in \\ students of Psychology
}

Henry Alex Flores Chacón

Universidad Continental

\section{RESUMEN}

Objetivos: Determinar la toma de conciencia de la identificación proyectiva en la relación con "el otro", en los estudiantes de Psicología de la Universidad Continental, al discutir un material clínico antes y después de su participación en una dinámica grupal. Métodos: Investigación descriptiva, de diseño cualitativo-estudio de caso. La recolección de datos fue mediante la entrevista y la observación psicológica. Los contenidos manifiestos de los estudiantes fueron registrados al discutir en grupo el material clínico de una sesión de psicoterapia de una paciente, presentado por escrito antes y después de su participación en la dinámica 12 sesiones). Resultados: Se determinó que la toma de conciencia de la identificación proyectiva en la relación con "el otro" en los estudiantes de Psicología, al discutir un mismo material clínico antes y después de la dinámica grupal muestra diferencias, tres contenidos manifiestos antes y 22 después de la dinámica. Conclusiones: La toma de conciencia de la identificación proyectiva en la relación con "el otro", es capaz de mostrar cambios favorables gracias a una dinámica grupal; logro que permite una comprensión sensible y empática de los pacientes. Considerar en la formación del estudiante de Psicología, un proceso así, fortalece su salud mental, aspecto que deviene en un logro congruente entre el desarrollo personal alcanzado y los conocimientos adquiridos.

Palabras claves: Identificación proyectiva, dinámica grupal, estudiantes de Psicología, psicoanálisis, insight.

1 Licenciado en Psicología, docente y supervisor del Círculo de Psicoanálisis de la Universidad Continental, candidato a psicoanalista del Instituto de la Sociedad Peruana de Psicoanálisis. 


\section{ABSTRACT}

Objectives: To determine the awareness of the prospective identification in relation to "the other", at discussing the clinical material before and after their participation in a group dynamic, with students in the area of Psychology at Universidad Continental. Methods: The research design was a case study, qualitative and descriptive. The data collection was carried out through the interview and the psychological observation. The students' contenidos manifiestos / reports were registered at the moment of discussing in group the clinical material from a patient's psychotherapy session and presented by writing before and after their participation in the dynamic (12 sessions). Results: It was determined that the awareness in the prospective identification in relation to "the other" with students of Psychology, at discussing the same clinical material and after the group dynamic, indicate differences in the awareness: 3 contenidos manifiestos before and 22 after the dynamic. Conclusions: the awareness of the prospective identification in relation to "the other" is able to show favorable changes, thanks to a group dynamic. This achievement allows the patients' sensitive understanding and empathy. Thus, It should be considered this kind of activity in the student's formation of psychology because it consolidates their mental health by being consistent between their personal development and the acquired knowledge.

keywords: Dynamic group, prospective identification, students of Psychology, psychoanalysis and insight.

\section{INTRODUCCIÓN}

Durante su formación los estudiantes de Psicología muestran aprendizajes que les permiten describir y explicar algunos fenómenos psicológicos; sin embargo, también serias dificultades en el nivel de desarrollo de la conciencia, entre otros aspectos psicológicos indispendables para su desempeño como futuros psicólogos, que les imposibilita la comprensión profunda de un paciente, la capacidad de brindar el diagnóstico psicológico apropiado y en consecuencia la recomendación indicada, y mucho más en la intervención terapéutica, que exige cierto nivel de conciencia para encaminar el proceso.

Todo proceso encaminado desde la teoría y la técnica psicoterapéutica, dentro de un encuadre establecido, conlleva a determinados logros, que van desde la toma de conciencia de ciertos aspectos personales y que tienen una estrecha vinculación con la manera como uno se relaciona con los demás, hasta la resolución de ciertos conflictos emocionales que impiden el uso adecuado de una serie de recursos del yo.

Si las dinámicas grupales son enfocadas por el psicólogo dentro de un marco similar a la psicoterapia y con objetivos precisos, como por ejemplo ampliar los niveles de conciencia de ciertos fenómenos que intervienen en la distorsión de la otra persona ("el otro"), sea el caso de la Identificación proyectiva; entonces surge el siguiente problema: ¿̇Existirán diferencias en la toma de conciencia de la identificación proyectiva en la relación con "el otro" en los estudiantes de Psicología, al discutir un mismo material clínico antes y después de participar en una dinámica grupal?

El estudio busca, primero, determinar la toma de conciencia de la identificación proyectiva en la relación con "el otro", en los estudiantes de Psicología de la Universidad Continental, al discutir un material clínico antes y después de su participación en una dinámica grupal; y segundo, establecer las diferencias de dicha toma de conciencia.

Ocurre que en la vida cotidiana escuchamos diversos comentarios de las personas acerca del mal servicio que reciben de algunos profesionales de la salud. Los usuarios, en ocasiones, muestran su insatisfacción porque sienten que no han cubierto sus demandas ni expectativas.

Si pensamos esta situación dentro del marco formativo universitario del futuro profesional, podemos preguntarnos si la formación 
recibida en esta época, está contemplando adecuadamente el desarrollo de ciertas competencias ideales para el trabajo con personas que requieren del servicio.

La información recibida en pregrado no bastaría para desarrollar una adecuada relación con "el otro", en este caso el paciente; nuestra historia personal está en juego, así como una serie de fenómenos psicológicos que de manera defensiva nos protegen de nuestras ansiedades, pero al mismo tiempo nos enajenan.

Es el caso del mecanismo de la identificación proyectiva, que en una primera instancia juega un papel organizador de la psicología de la persona, pero en otras circunstancias juega un papel defensivo equilibrador, que coloca en la otra persona aspectos indeseables de uno mismo e identificándolos allí con facilidad, negando toda posibilidad de tener algo "similar".

Los profesionales de la salud se ven cada día tomados por este mecanismo, distorsionando a sus entrevistados, identificando ciertos aspectos personales no resueltos en sus pacientes y lo que es peor, tratándolos desde esta percepción, hasta llegar a malos tratos, como por ejemplo, no entender adecuadamente lo que está pasando la persona, dar indicaciones que les sería útil al psicólogo y no necesariamente al paciente, ser poco empático con lo que quiere decir el paciente, insensible a temas tan delicados y de importancia para la persona.

Mesa y Rojas (1), en su investigación "Actividad referencial de estudiantes de Psicología del campo clínico con enfoque psicoanalítico", encontraron al comparar los resultados de la misma muestra en tiempos diferentes, de los estudiantes de Psicología en formación, que no había variado la capacidad de vincular emociones con los códigos simbólicos verbales, que manifiesta que la formación recibida no facilitaba un desarrollo en la actividad referencial mencionada en la investigación.

Sandler (2) encuentra que durante nuestra vida diaria y de manera inconsciente, el mecanismo de la identificación proyectiva, entre otros, se está implementado para lograr la calma y el equilibrio de nuestro organismo, lo que quiere decir que este mecanismo actúa de manera natural, más allá de una condición psicopatológica.

Ogden (2) ha subrayado el papel de la identificación proyectiva como vía de lo que ocurre internamente en la Psicología de la persona a lo interpersonal. En el encuentro paciente-psicólogo, surgen una variedad de experiencias objetivas y subjetivas que afectan a ambas partes, la falta de conciencia de estos componentes personales y duales es materia de un resultado ineficiente o iatrogénico.

Racker (2) relaciona la identificación proyectiva con la contratransferencia $y$ menciona que frente a las proyecciones masivas de parte de los pacientes, se hace indispensable diferenciar entre una identificación concordante (aquella que sintoniza con el discurso del paciente) y una identificación complementaria (aquella que lleva a identificarse con algún objeto interno del paciente, pudiendo actuarlo.

Osorio (3), en su investigación "La dinámica vincular celos-infidelidad", menciona que la identificación proyectiva obliga a los psicoterapeutas a una mayor profundización y ello implica un mayor tiempo de trabajo.

Painceira (4) encuentra en su labor como psicoanalista que la auténtica preocupación por "el otro" solo es factible si la persona ha transitado adecuadamente por la posición depresiva en etapas muy tempranas de la infancia, caso contrario un proceso psicoterapéutico generaría el desarrollo de ciertas áreas inconclusas de la personalidad.

Gheiler (5) encuentra que desprenderse de una serie de obstáculos, encontrarnos con nuestra propia autonomía, nos hace responsables, y ello nos conlleva a la posibilidad de ser más creativos. Este desarrollo personal es logrado mediante la participación de espacios de autoconocimiento como talleres, dinámicas, grupos de crecimiento, terapias, etc. 
Lopera, Ramírez, Zuluaga y Ortiz (6), en su trabajo "El método analítico como método natural", mencionan sus relaciones con el método científico y explicitan su vinculación íntima con la ética, en tanto hábito que se da como resultado de la incorporación del mismo. Este método tendría una estrecha relación con la técnica para ampliar la conciencia.

Lewin (7), desde su experiencia en el trabajo con grupos, menciona que la dinámica de grupo trata de dar mayor autonomía al sujeto y de hacerlo más consciente de su implicación personal. Al permitir una comunicación más espontánea y una mejor participación en el trabajo común, favorece la puesta en juego de funcionamientos más flexibles y adaptados.

Anzieu (8) descubre la importancia de la técnica psicoanalítica en el desarrollo de la persona en un trabajo de grupo. El paciente al decir libremente todo aquello que se le viene a la mente y al expresarse con libertad en el grupo y cada quien escuchar lo que dicen los participantes, inclusive ellos mismos, genera la ampliación de la conciencia.

De Mijolla (7), al referirse a los logros de la dinámica grupal, menciona que esta permite que la persona desarrolle su capacidad de autoobservarse, de ser crítico consigo mismo, de que se percate de aspectos suyos que juegan un papel importante en la comprensión de las diversas dificultades de las relaciones interpersonales.

Ascorra (9), en su artículo "Cómo y para qué se ha instaurado el discurso de la maximización de beneficios en la esfera psicoterapéutica: Análisis de las evaluaciones de costo-beneficio", hace referencia a la distorsión que se da en algunos países acerca de lo que es en sí un proceso terapéutico, por razones de costo y esfuerzo, argumentan que los cambios y los niveles de desarrollo se realizan en corto tiempo, por lo tanto una serie de intervenciones son entendidas como procesos profundos sin serlo.

El estudio pretende contribuir con la sociedad y las instituciones universitarias en el establecimiento o mantenimiento de una formación adecuada, basada en el desarrollo humano, de los profesionales de la salud, responsables de atender a personas que por su condición padecen un grave estado de vunerabilidad emocional y necesitan especial atención. Busca cumplir el espíritu de la nueva Ley Universitaria, que establece entre sus fines "formar profesionales de alta calidad de manera integral y con pleno sentido de responsabilidad social de acuerdo a las necesidades del país" (artículo 6), y que entre sus funciones compromete a las universidades a "contribuir al desarrollo humano" (artículo 7).

Las limitaciones estuvieron en el trabajo propiamente con el grupo y en los alcances

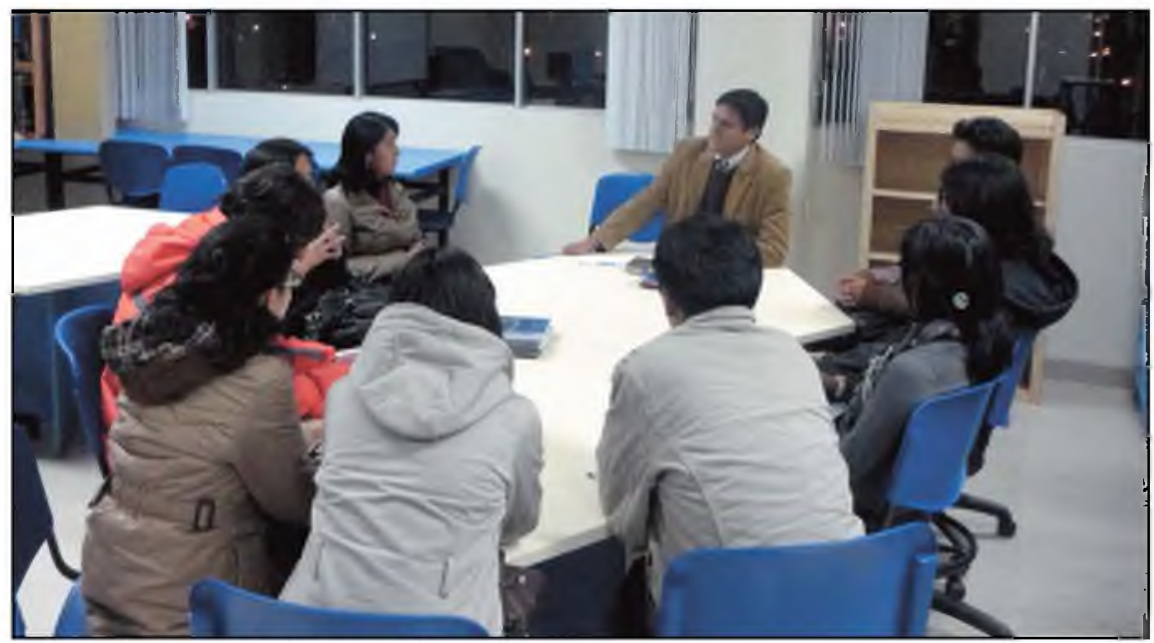

Figura $\mathrm{N}^{\circ}$ 1: Dinámica grupal. 
terapéuticos conseguidos. Los participantes de la investigación, al ser estudiantes de la casa de estudios y al estar siendo grabados en cada sesión de la dinámica grupal, hacía que se muestren en algunas sesiones algo reservados y tensos, situación que le restó espontaneidad y fluidez.

\section{MATERIAL Y MÉTODOS}

La muestra estuvo representada por un grupo de 12 estudiantes, 5 mujeres y 7 hombres de la Carrera de Psicología de la Universidad Continental, que se encontraban en su condición de practicantes relacionándose con pacientes, en el marco de prácticas pre profesionales que la institución les plantea como requisito antes de su internado o por prácticas pertenecientes a lo establecido por un curso determinado (figura $\mathrm{N}^{\circ} 1$ ).

La selección de la muestra fue de tipo no probabilístico e intencionado.

La investigación de alcance descriptivo, permitió conocer los contenidos manifiestos y latentes de los participantes, con respecto al análisis grupal de una sesión de psicoterapia de una paciente presentada por el investigador, tanto antes y después de la dinámica grupal orientada psicoanalíticamente.

La recolección de datos fue mediante la entrevista y la observación psicológica, antes y después de la dinámica, y fue grabada de manera audiovisual por un equipo manejado por un asistente. Estos datos fueron clasificados como contenidos que indican la toma de conciencia de la identificación proyectiva según definen los autores a tal mecanismo.

Los criterios que determinaron cómo hacer la clasificación, así como el análisis de los mismos, fueron determinados por el investigador haciendo uso del marco teórico y técnico del psicoanálisis. Después se contabilizaron los contenidos para la toma de conciencia antes y después de la dinámica, y pudo establecerse así un número de contenidos para lo que se planteaba cada objetivo y luego pasar a determinar una diferencia entre un antes y un después.

El nivel de confianza manejó el criterio de "credibilidad" como lo dice Salgado (10), es decir una investigación de tipo cualitativo

Tabla $N^{\circ}$ 1: Contenidos manifiestos en la discusión clínica antes de participar en la dinámica grupal.

\begin{tabular}{lc}
\hline \multicolumn{1}{c}{ Contenidos $\left(^{*}\right.$ ) } & Número de \\
\hline contenido & $\begin{array}{c}\text { Cantidad de } \\
\text { contenidos }\end{array}$ \\
\hline $\begin{array}{l}\text { Discriminación de contenidos internos personales (crisis y conciencia de identidad) } \\
\text { evocados a partir del material expresado por la paciente }\end{array}$ & 19,20 \\
$\begin{array}{l}\text { Capacidad para darse cuenta del actuar egocéntrico en la interacción con el otro } \\
\text { como preservación de los objetos buenos. }\end{array}$ & 00 \\
$\begin{array}{l}\text { Los fenómenos que experimenta el terapeuta (contratransferencia, empatía, } \\
\text { identificaciones, etc.) y la instrumentalización de los mismos en intervenciones a } \\
\text { favor de la toma de conciencia. }\end{array}$ & 00 \\
$\begin{array}{l}\text { Actitud comprensiva hacia el otro, desde el reconocimiento de aspectos indeseables } \\
\text { de uno mismo. }\end{array}$ & 00 \\
$\begin{array}{l}\text { ldentificación y reconocimiento de la tendencia a culpar a los demás como defensa. } \\
\text { ldentificación y reconocimiento de la actitud crítica del sujeto hacía ciertos aspectos } \\
\text { del otro. }\end{array}$ & 00 \\
$\begin{array}{l}\text { Aceptación responsable de ciertos contenidos personales motivadores del contenido } \\
\text { de un comentario personal acerca del otro. }\end{array}$ & 00 \\
$\begin{array}{l}\text { Reconocimiento del otro como objeto bueno también. } \\
\text { Subtotall }\end{array}$ & 00 \\
\hline Total de contenidos & 00 \\
\hline
\end{tabular}

$\left(^{*}\right)$ Los contenidos que se mencionan en la tabla son la integración y síntesis de los contenidos manifiestos hechos por los mismos participantes y que el investigador los agrupó por su similitud entre uno y otro. 
toma criterios de confianza como el entrenamiento y la disciplina subjetiva del investigador, la supervisión continua de los datos con un profesional experto en el tema, la contrastación de observaciones con un asistente y el uso de medios audiovisuales, que fueron el caso del presente trabajo.

\section{RESULTADOS}

La tabla $\mathrm{N}^{\circ} 1$ muestra que de 32 contenidos manifiestos en total, 3 mostraron la toma de conciencia de la identificación proyectiva en la relación con "el otro", en los estudiantes de Psicología de la Universidad Continental, al discutir un material clínico antes de su participación en una dinámica grupal, cantidad que representa el 9,4\% del total. contenidos manifiestos en total, 22 mostraron la toma de conciencia de la identificación proyectiva en la relación con "el otro", en los estudiantes de Psicología de la Universidad Continental, al discutir un material clínico después de su participación en una dinámica grupal, representando el $68,75 \%$ del total.

La tabla $N^{\circ} 3$ nos muestra la diferencia de la toma de conciencia de la identificación proyectiva en la relación con "el otro", en los estudiantes de Psicología de la universidad Continental, al discutir un material clínico antes, representado por 3 contenidos manifiestos y después, representado por 22 contenidos manifiestos, de su participación en una dinámica grupal, al realizar una resta simple, obteniéndose la cifra de 19.

La tabla $N^{\circ} 2$ considera que de 32

Tabla $N^{\circ}$ 2: Contenidos manifiestos en la discusión clínica después de participar en la dinámica grupal.

\begin{tabular}{|c|c|c|}
\hline Contenidos $\left({ }^{*}\right)$ & $\begin{array}{l}\text { Número de } \\
\text { contenido }\end{array}$ & $\begin{array}{l}\text { Cantidad de } \\
\text { contenidos }\end{array}$ \\
\hline $\begin{array}{l}\text { Discriminación de contenidos internos personales (crisis y conciencia de identidad) } \\
\text { evocados a partir del material expresado por la paciente }\end{array}$ & $\begin{array}{l}1,3,8,24,25 \\
28,30,32\end{array}$ & 08 \\
\hline $\begin{array}{l}\text { Capacidad para darse cuenta del actuar egocéntrico en la interacción con el otro } \\
\text { como preservación de los objetos buenos. }\end{array}$ & 2,23 & 02 \\
\hline $\begin{array}{l}\text { Los fenómenos que experimenta el terapeuta (contratransferencia, empatía, } \\
\text { identificaciones, etc.) y la instrumentalización de los mismos en intervenciones a } \\
\text { favor de la toma de conciencia. }\end{array}$ & $4,9,15,31$ & 04 \\
\hline $\begin{array}{l}\text { Actitud comprensiva hacia el otro, desde el reconocimiento de aspectos indeseables } \\
\text { de uno mismo. }\end{array}$ & 5 & 01 \\
\hline Identificación y reconocimiento de la tendencia a culpar a los demás como defensa. & 6,18 & 02 \\
\hline $\begin{array}{l}\text { Identificación y reconocimiento de la actitud crítica del sujeto hacía ciertos aspectos } \\
\text { del otro. }\end{array}$ & 7,22 & 02 \\
\hline $\begin{array}{l}\text { Aceptación responsable de ciertos contenidos personales motivadores del contenido } \\
\text { de un comentario personal acerca del otro. }\end{array}$ & 26 & 01 \\
\hline Reconocimiento del otro como objeto bueno también. & 27,29 & 02 \\
\hline Subtotal & & 22 \\
\hline Total de contenidos & & 32 \\
\hline Porcentaje sub total & & $68,75 \%$ \\
\hline \multicolumn{3}{|c|}{$\begin{array}{l}\text { (*) Los contenidos que se mencionan en la tabla son la integración y síntesis de los contenidos manifiestos hechos por los mismos } \\
\text { participantes y que el investigador los agrupó por su similitud entre uno y otro. }\end{array}$} \\
\hline \multicolumn{3}{|c|}{$\begin{array}{l}\text { Tabla } N^{\circ} \text { 3: Diferencia entre los contenidos manifiestos en la discusión clínica antes y después de } \\
\text { participar en la dinámica grupal. }\end{array}$} \\
\hline $\begin{array}{l}\text { Cifra del número de contenidos que mostraron la toma de conciencia } \\
\text { de la identificación proyectiva antes de la dinámica grupal }\end{array}$ & Resta & \\
\hline $\begin{array}{l}\text { Cifra del número de contenidos que mostraron la toma de conciencia } \\
\text { de la identificación proyectiva, después de la dinámica grupal }\end{array}$ & $22-03=$ & 19 \\
\hline
\end{tabular}




\section{DISCUSIÓN}

La investigación encuentra que si existen diferencias en la toma de conciencia de la identificación proyectiva en la relación con "el otro", en los estudiantes de Psicología de la Universidad Continental, al discutir un material clínico antes y después de su participación en una dinámica grupal.

Mesa y Rojas (1), en su investigación "Actividad referencial de estudiantes de Psicología del campo clínico con enfoque psicoanalítico", encontraron que no había variado la capacidad de vincular emociones con los códigos simbólicos verbales, dentro de la formación académica regular de los estudiantes; si bien es cierto en comparación a nuestra investigación los componentes psicológicos en estudio son diferentes, lo que si se podría afirmar a partir de los resultados hallados antes de la participación de los estudiantes en la dinámica grupal, es que hasta entonces la formación recibida no había facilitado la toma de conciencia de la identificación proyectiva en la relación con "el otro" (aún asistiendo estudiantes de ciclos superiores) y que ésta es tomada en cuenta luego no de una clase, sino, de un proceso de dinámica grupal orientada psicoanalíticamente; lo cual nos podemos plantear que de no haber ocurrido la dinámica, es bastante probable que al igual que los estudiantes de la investigación citada, no haya habido cambios al respecto.

Sandler (2) plantea que el mecanismo de la identificación proyectiva está presente en la normalidad y no necesariamente en la psicopatología; en comparación con nuestra investigación la podemos observar en la presencia natural y automática que se dio durante la discusión clínica, así mismo en la presencia de algunos contenidos manifiestos por los participantes pero que precisamente no generaban una distorsión severa, sino por el contrario una forma de equilibrio a sus apreciaciones.

Ogden (2), plantea que aquellos contenidos psicológicos internos, se expresan en las relaciones interpersonales, siendo así, afirma que algunas de las recomendaciones que damos a los pacientes están erradas; en nuestros hallazgos observamos comentarios, indicaciones y posibles consejos que le dirian a la paciente, bastante tomados por ocurrencias personales de los participantes, ausentes de empatía y comprensión objetiva, como por ejemplo: que es la culpable de todo o que es una dependiente sin entenderlo en un contexto de interrelación con los demás.

Racker (2) establece la diferencia entre una identificación concordante y una identificación complementaria con el paciente; es decir, es posible que podamos seguir adecuadamente la ilación de lo que nos dice el paciente, como que también podamos identificarnos con algún objeto interno de la otra persona, con la imagen del padre o de la madre persecutoria por ejemplo y desde ahí actuar como tales y ya no con nuestro criterio personal. Muchos de los participantes mostraron identificaciones con aspectos punitivos y sádicos de la paciente, anulando su actitud analítica y pensante sin conciencia.

Osorio (3) estima que el trabajo más profundo de la identificación proyectiva requiere de tiempo; nuestros resultados obtenidos lograron una toma de conciencia más a un nivel racional y de control consciente, eso no asegura un cambio profundo, eso requeriría de más tiempo, muchos aspectos psicológicos de los participantes aún estarían tomados por la identificación proyectiva.

Painceira (4), encuentra que la auténtica "preocupación por el otro" depende de la elaboración de la posición depresiva, es decir a partir de que surja el deseo de reparación del "otro" por haberlo agredido sea en la realidad o en la fantasía; al comparar con nuestros hallazgos, observamos que esto en un proceso tan breve de 12 sesiones, alcanza a surgir minimamente una actitud de preocupación por el otro, más no es algo tan elaborado y profundo como lo es toda una postura que integra el sentir, el pensamiento y la acción. consolidar ello, en el caso que se requiera, consideramos que 
se necesita de un tiempo mayor.

Gheiler (5) menciona que la libertad para ser creativos y responsables se logra al ser autónomos y al vencer obstáculos; en comparación con nuestra investigación observamos en los participantes que la toma de conciencia se incrementó en la medida de que se hacían responsables de sus propios contenidos internos, vencían obstáculos como el darse cuenta que algunas experiencias vividas habían determinado su forma de actuar y funcionaban de manera rígida sin posibilidad de hacer uso de la creatividad para pensar de otro modo las cosas del "otro", en este caso del paciente.

Lopera, Ramírez, Zuluaga y Ortiz (6), en su trabajo "El método analítico como método natural", plantean que el método analítico es factible de volverse un hábito en la medida que éste se incorpore; al comparar con nuestra investigación encontramos que algunos de los participantes habían desarrollando una actitud prudente antes de opinar del paciente, es como si, se detenían a pensar y a analizar lo que iban a decir, si es realmente una opinión objetiva basada en el paciente o en las vivencias personales del participante.

Lewin (7) menciona que las condiciones de libre comunicación en el grupo favorecen el desarrollo de la conciencia en lo que respecta a su implicación personal del sujeto; al comparar con nuestra investigación encontramos que las situaciones de libertad, en donde no se le decía al grupo que decir o no se descalificaba un tema que aparecía espontáneamente, traía consigo que se ocupen más de ellos o a que se percaten de lo que estaban diciendo, esto favoreció a la toma de conciencia de la identificación proyectiva, no por explicación del mecanismo, sino porque, surgía la conciencia de lo suyo propio involucrado en sus comentarios, al referirse sobre la otra persona.

Anzieu (8) plantea algo similar a lo anterior, con respecto a la libertad para comunicarse y la ampliación de la conciencia, pero agrega algo interesante, la escucha a los otros, incrementa también la conciencia; Si comparamos con nuestros resultados, la toma de conciencia ocurrió al escuchar a los otros cuando se referían de la paciente - cuando construían y creaban algo sobre ella, dentro de una dinámica. Es como sí algo se iluminara en medio de algo que estaba oscuro, cobraba sentido algo dentro de su persona a partir de lo que escuchó de un compañero.

De Mijolla (7), al referirse a los logros de la dinámica grupal, menciona a la capacidad de autoobservarse; en nuestra investigación podemos entender que la toma de conciencia de la identificación proyectiva, en términos de darse cuenta de aquellos contenidos que se supone están referidos a la paciente y que son nuestros, los participantes de la dinámica grupal fueron avanzando en la posibilidad de autoobservarse, uno de ellos dijo, en la discusión clínica después de la dinámica, que la primera vez fue bastante duro al criticar a la paciente acerca de su procedimiento para con su esposo y que se había percatado de que estaba aplicando la misma dureza, con que se trató así mismo cuando estuvo en una situación similar y que nunca se perdonó por ello.

Ascorra (9) advierte acerca de la importancia de entender que un proceso de cambio profundo y eficiente requiere de mucho tiempo y que no por razones económicas, politicas o de otro interés debemos confundir sobre ello, diciendo que es corto el tiempo; Nos queda claro que nuestra investigación ha conseguido logros a nivel racional con algunos cambios a nivel actitudinal y que es bastante probable que se repita algún procedimiento poco conciente, donde se termine usando nuevamente el mecanismo de la identificación proyectiva, no solo por que funciona de manera normal, sino por que se activa en algunas personalidades más que otras para protegerse de alguna ansiedad. La ampliación de conciencia en una mayor plenitud requiere de una dinámica más amplia.

Fue posible la toma de conciencia de la identificación proyectiva en la relación con "el otro", en los estudiantes de Psicología 
de la Universidad Continental al discutir un material clínico, después que participaron de la dinámica grupal orientada psicoanalíticamente; incrementando de ese modo cierta actitud de mayor sensibilidad y empatía al comprender a una paciente, pudiendo discriminar en cierta medida algunos aspectos personales de los de la paciente, apuntando a una objetividad en el conocimiento del "otro".

\section{Agradecimientos:}

A la psicóloga Isabel Mendoza por su valiosa colaboración en el proceso de la dinámica grupal.

\section{REFERENCIAS BIBLIOGRÁFICAS}

1. Mesa A, Rojas L. Actividad referencial de estudiantes de Psicología del campo clínico con enfoque Psicoanalítico. Colombia: Pensamiento Psicológico; 2006; 2(6): 61-76.

2. Sandler J. Proyección, Identificación, identificación proyectiva. Madrid: Tecnipublicaciones; 1989.

3. Osorio J. La dinámica vincular celosinfidelidad (identificación proyectiva). Colombia: Pensamiento Psicológico; 2011 ; 9(17): 97-102.

4. Painceira $A$. Repensando el psicoanálisis desde la persona. Buenos Aires: Lumen; 2007.

5. Gheiler M. La educación desde el Psicoanálisis. Lima: UPC; 2012.

6. Lopera J, Ramírez C, Zuluaga M, Ortiz J. El método analítico como método natural. Nómadas: Revista crítica de ciencias sociales y jurídicas. 2010; (25): 327-353.

7. De Mijolla A, Mijolla S. Fundamentos del psicoanálisis. Madrid: Síntesis; 2003.

8. Anzieu D, Martin J. La dinámica de los grupos pequeños. Madrid: Biblioteca Nueva; 2007.

9. Ascorra P Cómo y para qué se ha instaurado el discurso de la maximización de beneficios en la esfera psicoterapéutica: análisis de las evaluaciones de costo-beneficio. psicoperspectivas. 2002; 1: 21-43.

10. Salgado C. Análisis comparativo de la metodología cuantitativa y cualitativa. Lima: USMP; 2000. 